\title{
Chatbot as a Tool for Knowledge Sharing in the Maintenance and Repair Processes
}

doi:10.2478/mape-2021-0045

Date of submission to the Editor: 08/2021

Date of acceptance by the Editor: 09/2021

MAPE 2021, volume 4, issue 1, pp. 499-508

\section{Katarzyna Mleczko}

ORCID ID: 0000-0002-8463-1429

Silesian University of Technology, Poland

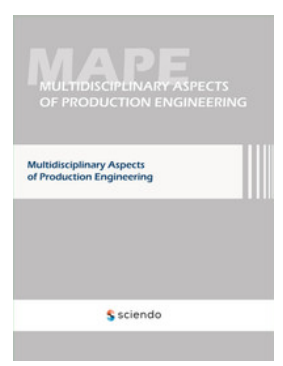

\section{INCRODUCTION}

Maintenance and repair operations are an activity that accompanies every user of any device. In addition to the activities carried out by professional maintenance services in industrial companies, it is important to consider the activities that are also carried out at home. Many devices of everyday use require some activities ensuring their proper functioning. There are also situations in which the ordinary user need to carry out minor repairs or eliminate defects intended by the manufacturer. This includes household appliances, radio and television equipment, and other devices that we use every day. Many manufacturers of household appliances anticipate minor faults or malfunctions in the equipment, but also recommend maintenance activities. These actions are to ensure that the equipment works as intended while maintaining its quality and functionality.

It should be noted that users of everyday equipment are very diverse in terms of many characteristics, such as age, gender, knowledge, experience, skills, and predispositions or abilities. These are the characteristics that may influence the way of performing maintenance activities, and thus the correctness and safety of use. Each manufacturer must provide instructions for use of the offered equipment and to inform the user about safety rules. These instructions are mainly provided in the traditional form (printed) in a few selected languages. However, in the era of digitalization and access to the Internet, users expect constant direct access to information via mobile devices (e.g. smartphones or tablets). Moreover, in addition to information on how to use the system, users are looking for various types of instructional videos, descriptions, or hints in graphical form based on the experience of other users. In most cases, this concerns situations related to the removal of emerging defects. The problem may be the quantity and quality of available materials.

We live in an era of rapid development of information technology, including those based on artificial intelligence. These solutions accompany us practically at every step - in cars, phones, in various types of media. So it seems reasonable 
to look for solutions based on artificial intelligence to support maintenance and repair activities carried out at home. One of such solutions is virtual assistants, which through communication similar to natural communication with the user can provide relevant knowledge resources while preventing misinformation. Virtual assistants (chatbots) are used primarily in the widely understood customer service of various online stores or service units (e.g. banks). Following this line, tools of this type can be used to support many repetitive service and repair activities by sharing customize knowledge.

\section{METHODOLOGY OF RESEARCH}

This paper is the result of test work on the application of a selected tool for creating chatbots based on artificial intelligence. The aim was to develop a tool to support the process of maintenance and repair of coffee maker at home. The recipient and the final user of the chatbot is an individual user - an adult person. The research methodology involved several stages, i.e.

Stage 1: Identification of needs and requirements concerning the created solution, including:

- Identifying the functionality of the SAP Conversational AI tool

- Developing user scenarios defining their needs,

- Selection of knowledge sources.

Stage 2: Building the knowledge base, including:

- Developing the structure of the knowledge base,

- Development of dialogue intentions,

- Development of expressions and context identifiers.

Stage 3: Designing the dialogue flow, including:

- Designing triggers,

- Assigning skills (generating responses).

Stage 4: Training And Testing.

Stage 5: Iterative refinement of dialogue flow and knowledge base content.

The research process is presented as a flow chart in Figure 1.

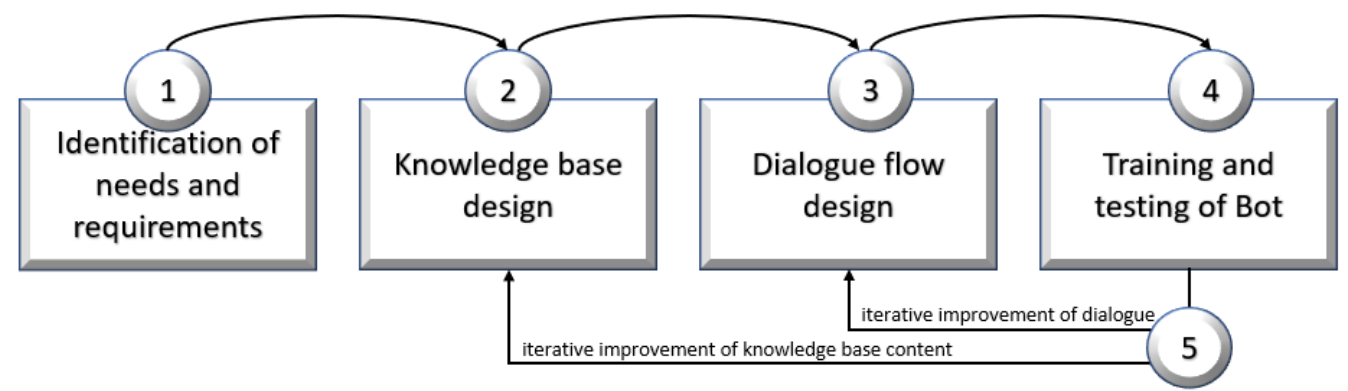

Source: own elaboration

Fig. 1 Process of Chatbot creation

\section{CHATBOT AS A TOOL BASED ON ARTIFICIAL INTELLIGENT}

Chatbots are computer programs that emulate a conversation with humans using text messages, navigation buttons, or using a simulated voice to provide 
a specific service (Sinarwati Mohamad Suhaili, et all., 2021). A characteristic of chatbots is that they communicate (communicate) with the user in a way that is very close to natural human speech (E. Kasthuri, S. Balaji, 2021). This is made possible by Natural Language Processing (NLP). NLP deals with the design and implementation of systems and algorithms capable of interacting through human language (Lauriola et all, 2021).

In the context of chatbots, by NLP we mean an artificial intelligence technology whose task is to analyze the utterances directed to a bot in terms of the actual intention of a given user (what does he want ?) and the parameters of this intention. NLP in chatbots is supported by learning algorithms using deep learning (machine learning) techniques. One of the tools for creating chatbots based on this functionality is SAP Conversational AI. It is a platform used to create chatbots, train them, connect with other systems and monitor their performance (https://cai.tools.sap). According to the manufacturer, SAP CAI, as a layer of the SAP Business Technology Platform, enables users to build and monitor intelligent chatbots in a single interface to automate tasks and workflows. Chatbots have found applications in many fields. They can be in the form of information tools, booking tools, marketing tools, recruitment tools supporting customer service, or supporting the completion of various types of forms. According to scientific sources, $80 \%$ of standard customer inquiries can be answered by a chatbot. An additional advantage of chatbots is their availability regardless of place and time, which is 24/7 (Chong T., 2021; Suthar S., 2020). These types of bots are called declarative, task-oriented chatbots, and they successfully serve as a comprehensive FAQ.

\section{DEVELOPMENT OF A CHATBOT SUPPORTING MAINTENANCE AND REPAIR OPERATIONS ON THE EXAMPLE OF A COFFEE MACHINE Identification of needs and requirements and building a knowledge base}

The idea of developing a chatbot to support maintenance and repair activities at home was to combine the functions of the advisory system, consultation with the authorized service, FAQ with the possibility of using natural communication of the user with the computer system.

The development of the chatbot began by defining a potential group of users and generating key functional characteristics. The selection of the object of research, which was a coffee machine, automatically determined the target group of users, which are individual customers, understood as adults. The object of service was a pressure coffee machine of one of the leading brands on the Polish market.

To determine the user-functional characteristics, the requirements description method used in software development according to agile methodology - user stories was used (Elallaoui M, 2018).

The description was done according to the recommended structure, i.e.

"As a [who?], I want to[what?], [so that]."

Examples of user stories are as follows: 
1. As a user of the coffee machine, I want to know how to take care of it so that it works properly.

2. As the machine operator, I want to know how to clean the machine for hygiene reasons.

3. 3) As a coffee machine user, I want to know what to do in case of a malfunction so I can quickly get rid of it without contacting an authorized service center.

4. As a coffee machine user, I want to know how to adjust settings to make my favorite coffee.

5. As the machine operator, I want to be able to recognize signals so that I can respond appropriately.

6. As a coffee machine user, I want to know what to do daily to make sure the coffee machine lasts as long as possible.

7. As the machine operator, I want electronic access to the user manual so that I do not have to search for it.

Based on user stories, the knowledge that the chatbot should use to provide answers to users was grouped.

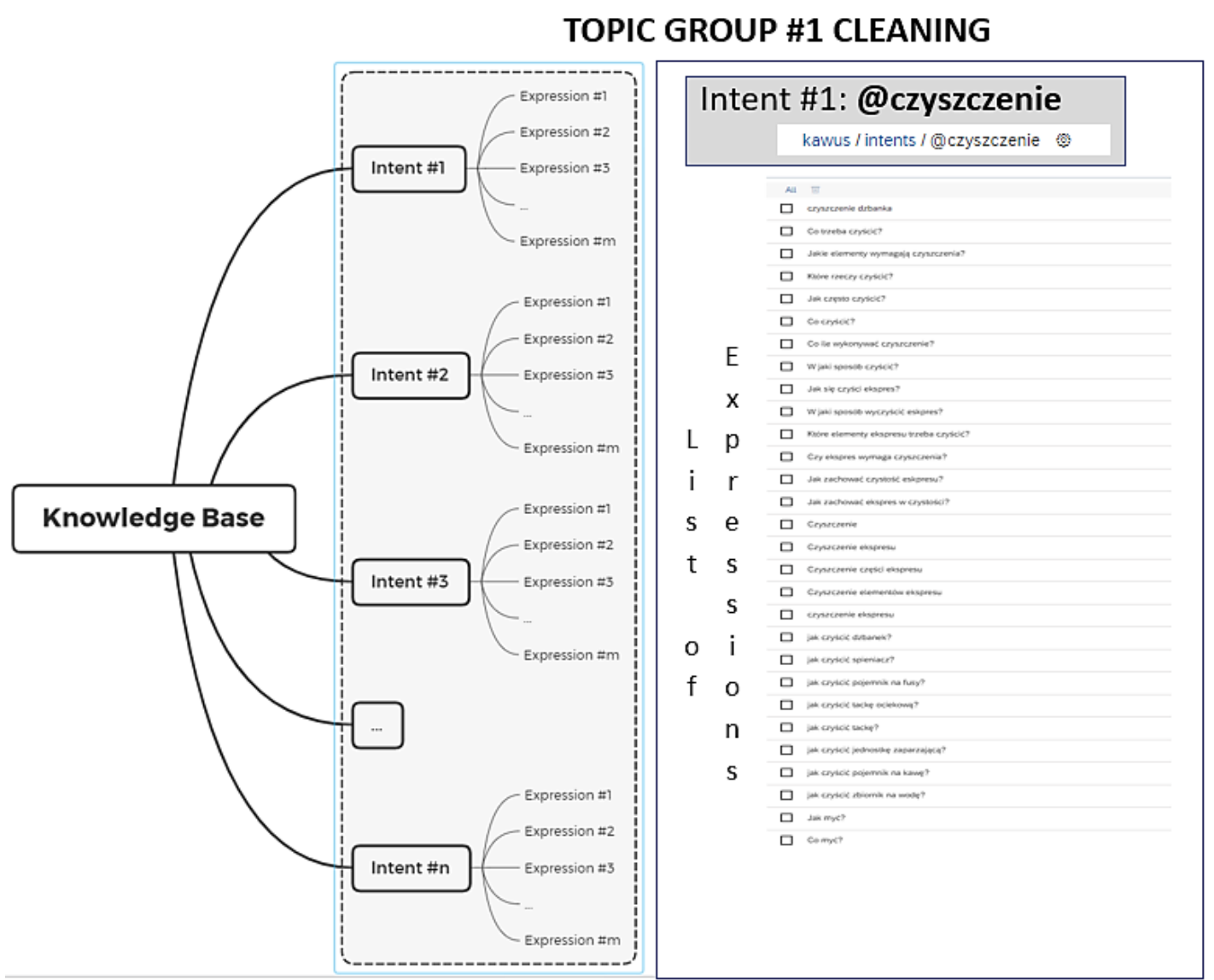

Fig. 2 Structure and an example of the relationship between knowledge base, intentions and expressions

Source: own elaboration

This was the basis for developing the structure of the knowledge base. It was determined that the main thematic areas that should be fed into the knowledge 
base are: day-to-day exploitation, maintenance, defect elimination. These are the areas that were of most interest to individual users, so the knowledge base should be built around them and in further stages expanded with the help of activity monitoring.

For each of the thematic groups, a group of intentions and expressions has been developed. Intentions are understood here as a separate thematic range, to which expressions are assigned. Expressions are written requests that can be uttered by the user during the dialogue with the chatbot. For a single intention, multiple expressions are assigned that can potentially be spoken (written, formulated) by the user. In other words, these are different forms of asking for the same thing. When building a list of expressions, it is a good idea to consider potential modes of utterance, newspeak, linguistic abbreviations, and linguistic or spelling errors.

The structure of the relationship between the knowledge base, intentions, and expressions is shown in Figure 2. Some standard intents are created to make the dialogue more natural. These are intentions, e.g., for greeting or ending a conversation or politeness phrases.

\section{Contents}

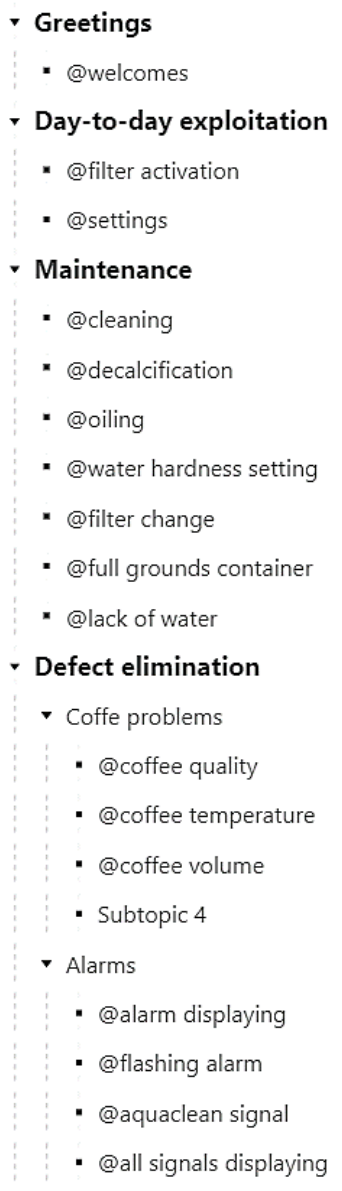

Fig. 3 The structure of the chatbot content

Source: own elaboration 
Figure 3 shows a summary of the generated intentions for a chatbot supporting the execution of maintenance and repair activities for a coffee machine.

It is important from the point of view of later dialogue design to extract context identifiers, the so-called Entities, from expressions. Entities allow you to detail and increase the recognizability of intentions and context detection. For example, for the defined intention @cleaning, entities are specified to indicate the items to be cleaned. These entities are, for example, the pot, brewing unit, coffee container, grounds container, frother, drip tray, and water tank. The more entities are extracted from expressions, the more detailed the context of the utterance can be interpreted and feedback can be built.

\section{DIALOGUE FLOW DESIGN}

Expressions are the basis of the dialogue between the user and the virtual assistant. Once the possible requests are known, it is necessary to "teach" the chatbot to respond to them. To do this, it is necessary to prepare the answers that will be given to the user after recognizing the expression and assigning it to an intention. In the SAP CAI tool, these are called skills.

The scenario of using a virtual assistant assumes that it will provide knowledge based on the documentation provided by the manufacturer, but also on external, reliable, and verified sources, such as contact with the authorized service or experiences of other users. It is assumed that as a result of dialogue the user will receive a condensed answer presented in various attractive and comprehensible forms. These may be text formats, graphics, or links. The set of possibilities is presented in Figure 4. The presented content is detailed and compact, but it presents procedures on a step-by-step basis.

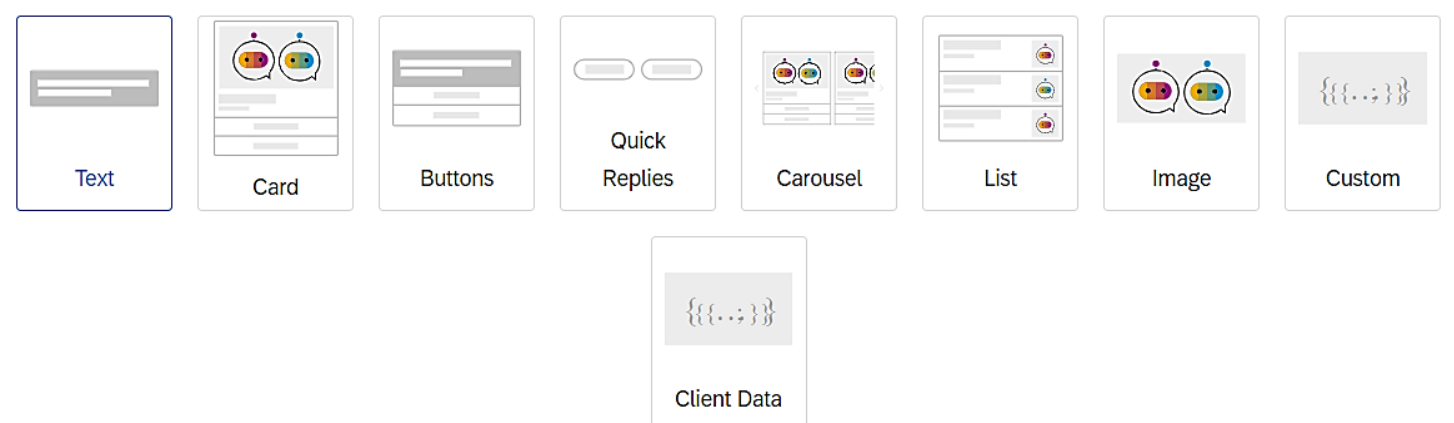

Fig. 3 Message Types - Screenshot from SAP CAI

The advantage of tools for building chatbots is the ability to design dialogue flow and configure triggers that trigger a response appropriate to a given (recognized) intention. Besides the intention itself, triggers can be context identifiers.

In advance, the designer assumes a certain flow of the conversation and assumes that if a phrase identifying the intention appears, plus a characteristic keyword, the customer (user) expects a specifically formulated response. To this 
end, triggers are designed using a previously developed knowledge base structure.

The structure of skill generation can be represented by a diagram:

If the user utters a phrase recognized as an intention and entities are detected then take an action.

At the same time, triggers can be combined using logical conjunctions such as "and", "or". The action is to display the ready feedback that is the focus of the user.

\section{BOT TRAINING AND TESTING}

At each stage of building a virtual assistant, its learning processes are supported. Among other things, the correctness of intention recognition and entity detection is tested, followed by the ability to generate responses.

An example of such a test is shown in Figure 4.

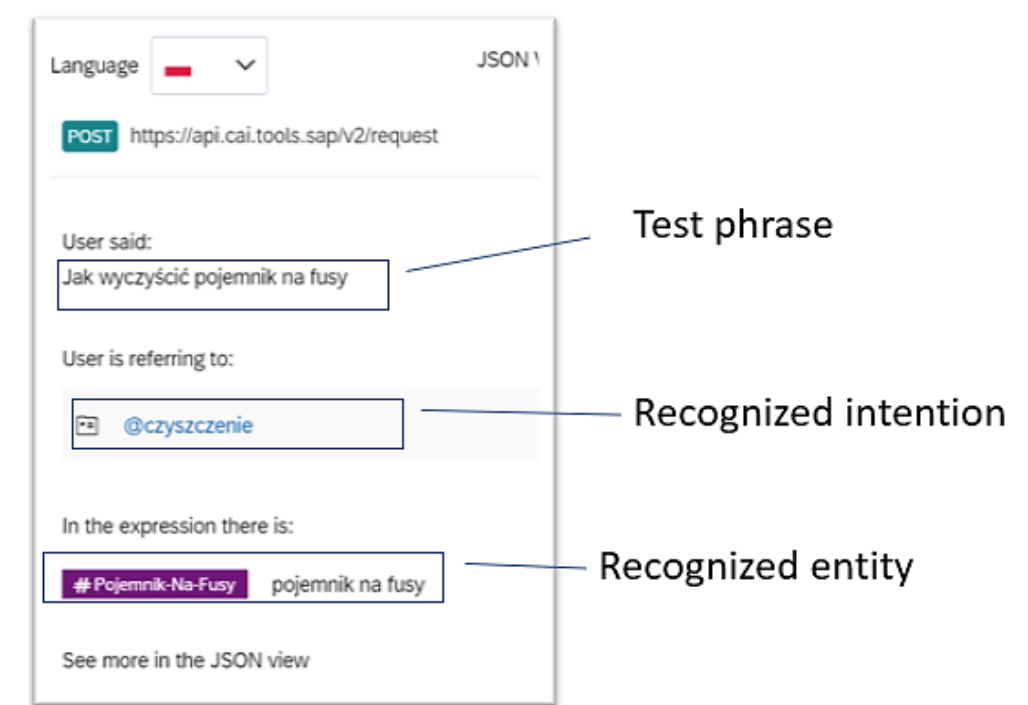

Fig. 4 Expression Analysis window - screenshot from SAP CAI

If there is any problem with intent recognition, the designer can manually teach the bot certain behaviors. Supervised learning is then used, which involves pointing to and remembering patterns. The designer has access to all sentences uttered by the user and can analyze which of the phrases have not been recognized by the program. If the reason is a lack of sentences in the knowledge base, it is improved.

\section{DIALOGUE EXAMPLE}

During the testing stage, the dialogue can be previewed in a specially dedicated window that looks like the final chatbot.

Example 1 (see Fig. 5) shows a correctly running dialogue with a virtual assistant: 


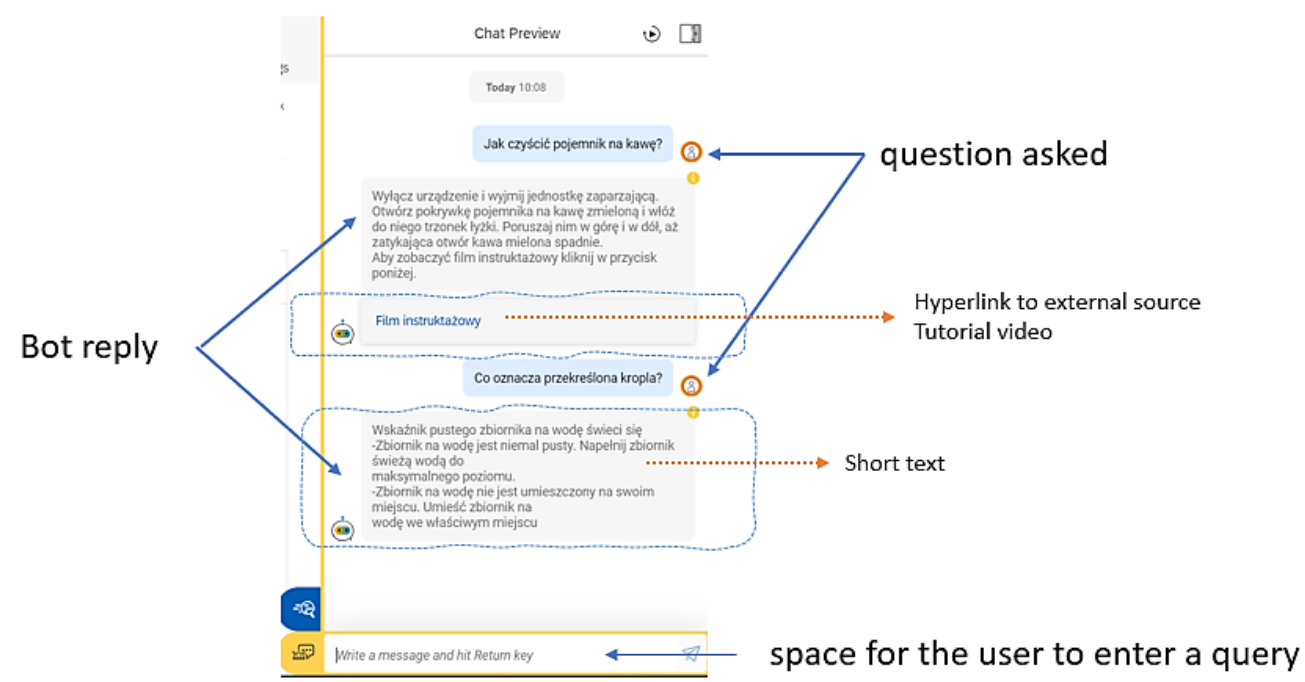

Fig. 5 Example of correctly dialogue flow

Source: own elaboration

The second example (see Fig. 6) shows a situation where the Bot has not correctly identified the trigger or has picked up too many of them at once. As a result, it cannot provide a substantive answer. As you can see, the user does not remain unresponsive, and the assistant continues the conversation by suggesting another way to ask the question or provides the full version of the user guide provided by the manufacturer.

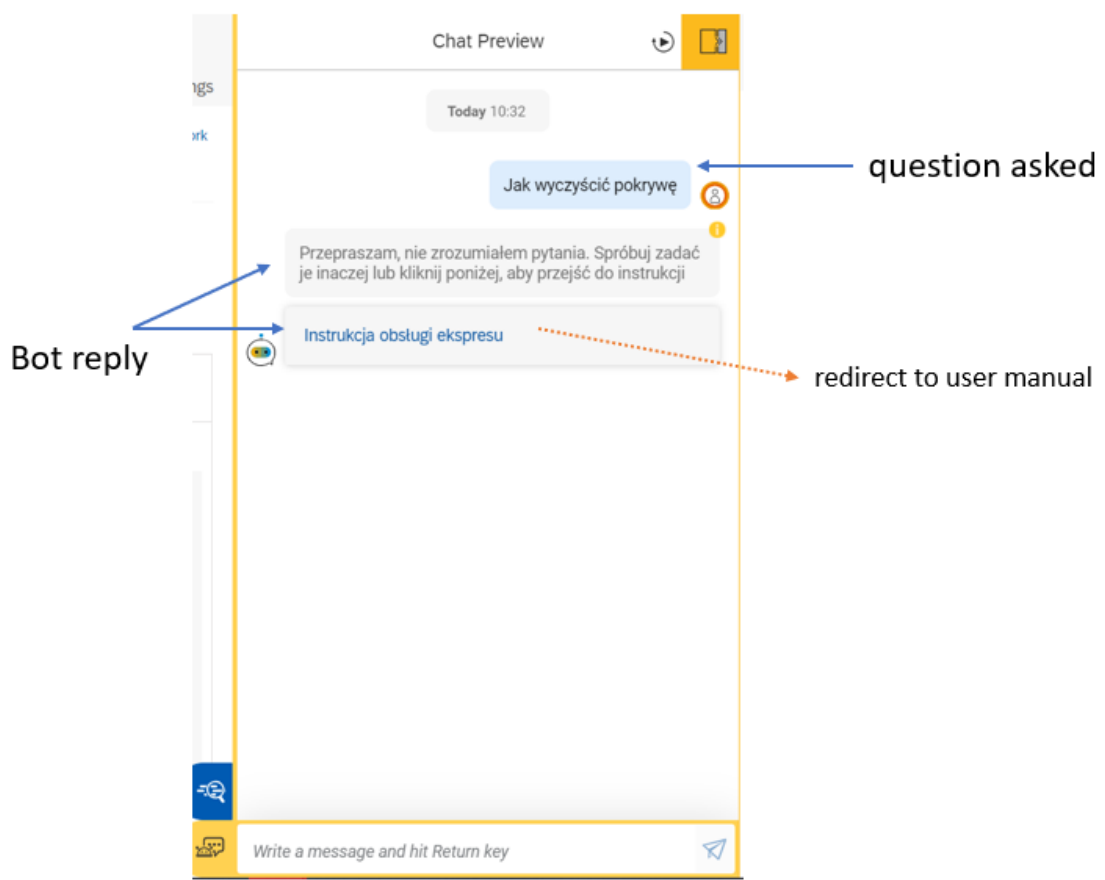

Source: own elaboration

Fig. 6 Example of not correctly dialogue flow

Each conversation between the chatbot and the users (not belonging to the project team) - serves to improve the knowledge base and the dialogue 
mechanisms. In general, the more interactions with users - the chatbot will get better and better by training mechanisms of its knowledge base.

\section{CONCLUSION}

The research shows that the use of modern technologies is not reserved only for industrial solutions, but can be applied in common use. Solutions based on artificial intelligence can provide solutions to many problems of the modern world. This is particularly visible in the era of challenges related to ecology (eg. minimization of paper consumption) and expectations of fast access to information regardless of place and time. Treating chatbots as a tool supporting the customer in current activities, quick problem-solving shows the potential and care for the image of the producers. Developing the functionality of virtual assistants used for marketing activities with the functionality of technical support can bring tangible benefits both in the economic and social spheres. It should be noted that electronic versions of documents, knowledge transferred in an interactive form can be easily updated and presented in different forms. This increases the attractiveness of transmitted knowledge, users can choose the appropriate form, and access to these resources becomes easier. The user is not forced to search for interesting issues in multi-page manuals, but instead receives precisely selected most relevant messages. Recognition of human speech and free conversation with a virtual assistant positively influences people's approach to this type of solution. In addition, chatbots based on artificial intelligence have predictive capabilities and are able to anticipate related service actions, determine the timing of these actions, and guide them step by step. Like any solution, chatbots are not without their drawbacks. Many people, given the choice of contact with another human being, will prefer it to contact with the most advanced artificial intelligence. The disadvantage of chatbots is also their underdevelopment and too many mistakes in interpretation of intentions and giving answers. However, it seems that treating a chatbot as a source of knowledge from multiple verified sources, delivered on a just-in-time basis, outweighs or at least compensates for its disadvantages.

\section{ACKNOWLEDGEMENTS}

This article is the result of research conducted at the Department of Production Engineering, Faculty of Organization and Management, Silesian University of Technology, within the statutory work project number 13/030/BK-21/0065.

\section{REFERENCES}

Chong Terrence, Yu Ting, Keeling Debbie Isobel, de Ruyter Ko, (2021). I-chatbots on the services frontline addressing the challenges and opportunities of agency, Journal of Retailing and Consumer Services, Volume 63, ISSN 0969-6989, https://doi.org/10.1016/j.jretconser.2021.102735.

Elallaoui Meryem, Nafil Khalid, Touahni Raja, (2018) Automatic Transformation of User Stories into UML Use Case Diagrams using NLP Techniques, Procedia Computer 
Science, Volume 130, pp. 42-49, ISSN 1877-0509, https://doi.org/10.1016/j.procs.2018.04.010.

Kasthuri E., Balaji S., (2021). Natural language processing and deep learning chatbot using long short term memory algorithm, Materials Today: Proceedings, ISSN 2214-7853, https://doi.org/10.1016/j.matpr.2021.04.154.

Lauriola I., Lavelli A. and Aiolli F., An introduction to Deep Learning in Natural Language Processing: Models, techniques, and tools, Neurocomputing, https://doi.org/10.1016/j.neucom.2021.05.103

SAP Conversational Al platform website, https://cai.tools.sap

Sinarwati Mohamad Suhaili, Naomie Salim, Mohamad Nazim Jambli, (2021). Service chatbots: A systematic review, Expert Systems with Applications, Volume 184, ISSN 0957-4174, https://doi.org/10.1016/j.eswa.2021.115461

Suthar, S., (2020). Top 10 Al-powered chatbots to skyrocket your sales. The Next Scoop. Available at: https://thenextscoop.com/ai-powered-chatbots-to-skyrocketsales/. (Accessed 28 February 2021).

\begin{abstract}
In the era of digitization and general access to Internet resources, it seems natural to expect fast, direct, and tailored access to the knowledge of interest. The research shows that the use of modern technologies is not reserved only for industrial solutions, but can be applied in common use. Solutions based on artificial intelligence can provide solutions to many problems of the modern world. This is particularly visible in the era of challenges related to ecology (e.g. minimization of paper consumption) and expectations of fast access to information regardless of place and time. Treating chatbots as a tool supporting the customer in current activities, quick problem-solving shows the potential and care for the image of the producers. Developing the functionality of virtual assistants used for marketing activities with the functionality of technical support can bring tangible benefits both in the economic and social spheres. It should be noted that electronic versions of documents, knowledge transferred in an interactive form can be easily updated and presented in different forms. This increases the attractiveness of transmitted knowledge, users can choose the appropriate form, and access to these resources becomes easier. Artificial intelligence-based tools in the form of chatbots (also called virtual assistants) seem to be a solution worthy of interest. This paper presents the process of developing a virtual assistant to support the maintenance and repair activities of small household appliances. A chatbot aims at gathering in one place and then providing dedicated knowledge resources to an individual user on a just-in-time basis.
\end{abstract}

Keywords: knowledge sharing, maintenance, chatbot, Al, virtual assistant 